\title{
Attentional Gradient for Crossmodal Proximal-Distal Tactile Cueing of Visual Spatial Attention
}

\author{
ROSLIZAWATY MOHD ROSLI, HONG Z. TAN, and ROBERT W. PROCTOR, Purdue University \\ ROB GRAY, Arizona State University
}

\begin{abstract}
Past studies have established a crossmodal spatial attentional link among vision, audition, and touch. The present study examined the dependence of visual attention on the distance between a distal visual target (a changing element among static distractors) and the quadrant of the visual display cued by a proximal tactile stimulus. The distance between the center of the cued visual quadrant and the visual target was one of six values: $0,90,180,350,450$, and 550 pixels. The distances of 0 , 90, and 180 corresponded to the valid tactile cueing condition, where the tactile cue and the visual target occurred in the same quadrant. The distances of 350,450 , and 550 corresponded to the invalid tactile cueing condition, where the tactilely-cued quadrant did not match that of the visual change. Results from 10 young adults showed that mean response time increased with respect to the cue-target distance, thereby confirming a gradient of visual attention for proximal-distal tactile cueing. In addition, the response times for valid tactile cues were shorter than those for invalid tactile cues, confirming earlier findings that valid tactile cues facilitate visual search and invalid tactile cues interfere with visual search. The findings of the present study have implications for the design of multimodal attention-cueing systems for practical applications such as collision warning systems in automobiles.
\end{abstract}

Categories and Subject Descriptors: H.1.2 [Models and Principles]: User/Machine Systems—Human information processing; H.5.1 [Information Interfaces and Presentation]: Multimedia Information Systems-Artificial, augmented, and virtual realities; H.5.2 [Information Interfaces and Presentation]: User Interfaces—Evaluation/methodology; Haptic I/O

General Terms: Design, Experimentation, Human Factors

Additional Key Words and Phrases: Attention gradient, crossmodal attention cueing, proximal-distal cueing, tactile cueing, eye gaze

ACM Reference Format:

Mohd Rosli, R., Tan, H. Z., Proctor, R. W., and Gray, R. 2011. Attentional gradient for crossmodal proximal-distal tactile cueing of visual spatial attention. ACM Trans. Appl. Percept. 8, 4, Article 23 (November 2011), 12 pages.

$\mathrm{DOI}=10.1145 / 2043603.2043605$ http://doi.acm.org/10.1145/2043603.2043605

This research was partially supported by a National Science Foundation Award Grant IIS-0533908.

A brief summary of this work was presented as part of a review paper at the Human Vision and Electronic Imaging XIV Conference [Tan et al. 2009].

Authors' addresses: R. Mohd Rosli and H. Z. Tan, Haptic Interface Research Laboratory, Purdue University, 465 Northwestern Av., West Lafayette, IN 47907-2035; email: \{rmohdros@gmail.com; hongtan@purdue.edu\}; R. W. Proctor, Human Performance Laboratory, Purdue University, 703 Third Street, West Lafayette, IN 47907; email: \{proctor@psych.purdue.edu\}; R. Gray, Perception and Action Laboratory, Arizona State University, Santa Catalina Hall, 7291 E Sonoran Arroyo Mall, Mesa, AZ 85212; email: \{robgray@asu.edu\}.

Permission to make digital or hard copies of part or all of this work for personal or classroom use is granted without fee provided that copies are not made or distributed for profit or commercial advantage and that copies show this notice on the first page or initial screen of a display along with the full citation. Copyrights for components of this work owned by others than ACM must be honored. Abstracting with credit is permitted. To copy otherwise, to republish, to post on servers, to redistribute to lists, or to use any component of this work in other works requires prior specific permission and/or a fee. Permissions may be requested from Publications Dept., ACM, Inc., 2 Penn Plaza, Suite 701, New York, NY 10121-0701 USA, fax +1 (212) 869-0481, or permissions@acm.org.

(C) 2011 ACM 1544-3558/2011/11-ART23 $\$ 10.00$

DOI 10.1145/2043603.2043605 http://doi.acm.org/10.1145/2043603.2043605

ACM Transactions on Applied Perception, Vol. 8, No. 4, Article 23, Publication date: November 2011. 


\section{INTRODUCTION}

The nature of visual attention has been the topic of considerable research in cognitive psychology and neuroscience (see, for example, Proctor and Read [2009]). The present study was motivated by a desire to understand how visual attention is distributed spatially when directed to a region by a cue. It is part of an ongoing research program investigating, among other things, the spatial and temporal parameters required for facilitatory multimodal interactions using visual, auditory, and tactile signals [Gray and Tan 2002; Ho et al. 2005; Jones et al. 2008]; see also Tan et al. [2009] for a recent review. The results of these studies provide the basic knowledge needed by designers of multimodal interfaces for various applications such as driver assistance and collision warnings.

One of the first and most basic questions is how close should the cueing signal be to the target event? Results from several experiments using visual cues and visual targets have shown that the facilitatory effects of attention on response performance (in terms of faster response times and improved sensitivity) decrease monotonically with increasing distance between the cue and target locations [Downing and Pinker 1985; Shulman et al. 1979; Van der Heijden 1992]. This is often referred to as the spotlight, or attentional gradient, effect [Posner et al. 1980; Föcker et al. 2010]. Neurological and physiological studies also demonstrate increased brain activity at areas associated with cues [Brefczynski and DeYoe 1999; Somers et al. 1999; Treue 2001; Assad 2003]. There is evidence that people can maintain multiple spotlights [McMains and Somers 2004] with high efficiency and without compromising performance levels [McMains and Somers 2005], although for many tasks attention will be focused on a single location. Furthermore, the spatial distribution of visual attention can be quite broad, with a monotonic decrease continuing up to cue-target separations as large as $20^{\circ}$ of visual angle [Shepherd and Müller 1989; Föcker et al. 2010]. Quantifying the extent of the gradient effect for various crossmodal cue-target pairs is important for informed design of attention cueing systems.

One limitation of early research on the distribution of attention is that it primarily considered only visual cueing of visual attention. This situation has, however, changed dramatically over the last several decades, in which a growing body of literature has demonstrated the effectiveness of crossmodal attention cueing for redirecting spatial attention to specific locations in the cases of vision and audition [Perrott et al. 1990]; vision and touch [van Erp et al. 2005; Jones et al. 2008; Van der Burg et al. 2009]; and among vision, audition, and touch [Spence et al. 1998; Ho et al. 2005]; see also Spence and Driver [2004], for a review. We are particularly interested in how attention is distributed across space when visual attention is reoriented by a tactile cue. Gray et al. [2009] studied the distribution of attention by comparing unimodal visual and crossmodal (tactile and auditory) cueing. Visual targets presented above or below the forearm were preceded by vibrotactile cues presented on the forearm, auditory cues presented below the forearm, or visual cues presented on the forearm. Unimodal visual cueing resulted in an attentional focus that was significantly narrower than that produced by crossmodal cues: the distribution of visual attention for visual cues had roughly half of the lateral extent of that produced by tactile cueing, and roughly one fourth of the lateral extent of that produced by auditory cueing (see Föcker et al. [2010], for similar results with auditory cueing). It was estimated that the spatial extents of the attentional gradient were $\pm 9^{\circ}, \pm 14^{\circ}$, and $\pm 17^{\circ}$ for the visual, tactile, and auditory cues, respectively.

This previous research (and similar studies looking at only audio-visual interactions by, for example, Driver and Spence [1998] and Schmitt et al. [2001]) examined crossmodal orienting when the targetcue separations were relatively small, so that the target was very near to the proximal surface on the skin that was stimulated by the tactile cue. Recently, it was shown that tactile cues can be used to reorient visual attention effectively for very large cue-target separations if a proximal surface on the body is mapped onto a set of distal locations in external space. For example, tactile cues presented to a user's back can be used to reorient visual attention to specific locations on a visual display in front of 
the user [Tan et al. 2003]. In another study, tactile cues presented to a driver's waist were shown to be effective at alerting impending collisions in front of or behind the vehicle [Ho et al. 2005; 2006]. The question that arises is whether proximal-distal attentional reorienting produces similar distribution of attention as proximal-proximal reorienting does.

The goal of the present study was to measure the distribution of visual attention following a tactile cue presented on the back. To our knowledge, this is the first quantitative study of attentional gradient using proximal-distal tactile cueing. Comparing this distribution to what we previously reported for proximal-proximal crossmodal reorienting [Gray et al. 2009] will help address the question of whether proximal-distal and proximal-proximal tactile cueing involve the same mechanism. If they do, then we would expect to see similar distributions of visual attention.

\section{METHODS}

\subsection{Participants}

Ten participants ( 5 females; ages of $20-35$ years, mean $=25$ years) took part in the experiment. Three participants received payment for their time. All had normal or corrected-to-normal vision and reported no known abnormalities with tactile perception on their backs. The participants gave their informed consent to the experimental procedures approved by the Institute Review Board at Purdue University.

\subsection{Apparatus and Stimuli}

The hardware consisted of a host computer, an eye-tracker system, an LCD visual display, a tactile back display, and a chinrest. The host computer ran a program that started and stopped the trials, generated the visual stimuli, and recorded participants' response times for each trial. The eye-tracker system consisted of an infrared-based eye-tracker (RK-726PCI Pupil/Corneal Reflection Tracking System, ISCAN Inc.) that recorded the eye movements made by participants in each trial at an update rate of $60 \mathrm{~Hz}$. The LCD visual display was placed $68 \mathrm{~cm}$ in front of the participant. At the viewing distance of $68 \mathrm{~cm}$, the display occupied a viewing angle of $\pm 10.6^{\circ}$ along its height $(25.4 \mathrm{~cm})$ and $\pm 14.0^{\circ}$ along its width $(33.8 \mathrm{~cm})$. The tactile back display delivered tactile cues to the back of the participant. The chin rest with a forehead stabilizer (Table Mounted Head Restraint, Applied Science Group, Inc.) was used to confine the movement of the participants' head.

The visual stimuli consisted of 40 rectangular white bars positioned horizontally or vertically on a black background (see Figure 1) with 10 bars per quadrant (see Figure 2). The dimension of each bar is 51 pixels $(\mathrm{L}) \times 19$ pixels $(\mathrm{W})$, or equivalently $1.7 \mathrm{~cm}(\mathrm{~L}) \times 0.6 \mathrm{~cm}(\mathrm{~W})$, or $1.43^{\circ} \times 0.51^{\circ}$, on the LCD monitor (76 dpi). The placement of the bars was random on each trial, with the constraint that no two white bars could overlap on each other. The visual change-detection task was based on the change blindness phenomena [Rensink et al. 1997]. It followed the flicker paradigm where two almost-identical visual scenes were displayed alternately with a blank screen inserted between scenes to eliminate motional cues (see Figure 1 in Rensink [2000]). In our case, the only difference between the two scenes was that one of the 40 bars changed its orientation from vertical to horizontal and vice versa. The participants' task was to detect and localize the bar that was changing orientation.

In our previous studies [Tan et al. 2003; Jones et al. 2008], the visual scene was divided into four quadrants, and visual targets were randomly selected to be in one of the four quadrants prior to each trial. The present study further required that the visual targets occur at one of six preset distances from the center of the tactilely-cued visual quadrant (see Figure 2). The six radii were 0, 90, 180, 350, 450 , and 550 pixels from the center of the tactilely-cued quadrant. Three of the radii $(0,90$, and 180$)$ lay within the cued quadrant, and therefore were used for the trials where the tactile-cue quadrant matched the visual-change quadrant (i.e., valid cueing). The remaining three radii of 350,450 , or 550 


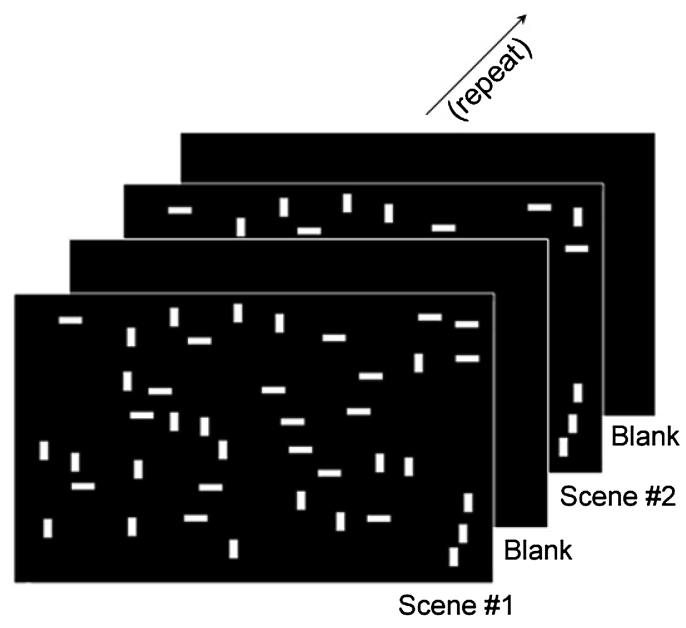

Fig. 1. The sequence of visual stimuli for the change detection task in this study. The two visual scenes differ in the orientation of one of its elements (the upper-right bar in the example shown here). The sequence continued until the participant clicked the mouse button to indicate the detection of the changing element.

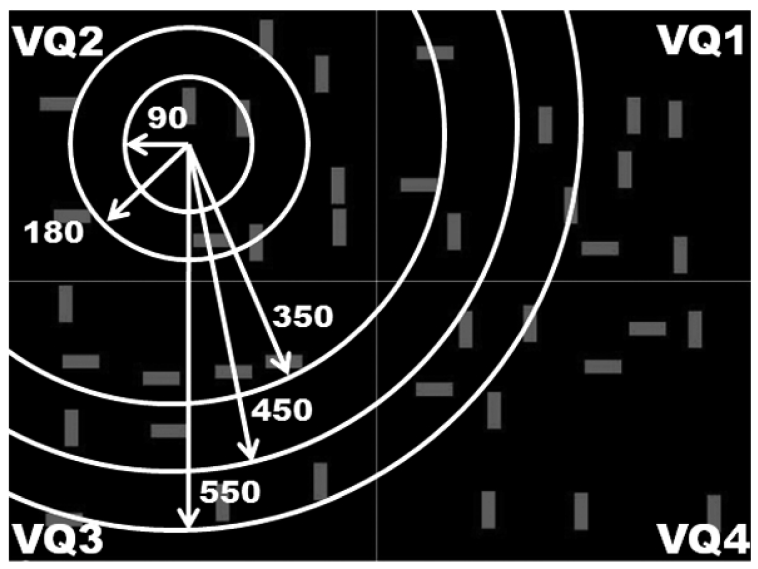

Fig. 2. The placement of the visual targets. Shown in the background are the 40 rectangular bars equally distributed in the four visual quadrants (VQ1, VQ2, VQ3, and VQ4). The circles/arcs indicate where targets could occur for the example where VQ2 was cued tactilely; they correspond to the following cue-target distances in pixels (and degrees): $0\left(0^{\circ}\right), 90\left(2.53^{\circ}\right), 180\left(5.06^{\circ}\right)$, $350\left(9.76^{\circ}\right), 450\left(12.47^{\circ}\right)$, and $550\left(15.13^{\circ}\right)$, respectively. During the experiment, the participant only saw the 40 horizontal and vertical bars on a dark background.

were not in the cued quadrant and were used for trials where the tactile cues were invalid (i.e., the tactile-cue quadrant did not match the visual quadrant where change occurred).

The tactile back display consisted of the four corner tactors aligned in a $2 \times 2$ array occupying an area of $20 \mathrm{~cm}(\mathrm{~W}) \times 15 \mathrm{~cm}(\mathrm{H})$. Each tactor (VBW32, Audiological Engineering Corp.) could be independently driven with a $60-\mathrm{ms} 290-\mathrm{Hz}$ sinusoidal pulse delivered by a custom-designed controller box (Haptics Interface Research Laboratory at Purdue University). The four tactor locations corresponded to the four visual quadrants on the computer monitor. Vibration intensity was adjusted so that the vibrations could be felt clearly through whatever clothing the participants happened to be wearing. 


\subsection{Procedure}

The session for each participant began with a tactile-cue localization task, followed by eye-tracker system calibration. The tactile-cue localization task consisted of 10 trials. At each trial, one randomlyselected tactor was turned on and the participant was asked to click one of four large buttons in the center of the four visual quadrants to indicate which tactor was felt. The tactor localization task was performed to ensure that the participants could correctly localize the four tactors. The eye-tracker calibration used a 9-point calibration procedure, where the participants followed a dot that moved along nine locations consisting of the four corner points on the computer monitor, the four mid-points along the four edges of the monitor and the center of the monitor. The eye-tracker was calibrated for each individual participant at the beginning of each experimental session and after each break during the experiment.

Each participant performed 15 40-trial experimental runs. The participants were instructed to search the visual scene until they found the bar that was changing orientation. Three of the 15 runs were performed without tactile cues to obtain baseline performance measures. The remaining 12 runs were performed with tactile cues. The three baseline runs were interspersed among the 12 tactilecueing runs in random order for each participant. The validity of the tactile cues was fixed at $75 \%$, meaning that on $75 \%$ of the trials, the tactilely-cued quadrant matched the quadrant where the visual target appeared. The participants were encouraged to use the tactile cue to aid their visual search. A 30-trial practice run was conducted prior to the main experiment, but the data was not analyzed.

At each trial, the following parameters were randomly selected: tactile-cue location, valid vs. invalid cueing, and visual-target location. The tactile cue was delivered by one of the four corner tactors on the back, with equal a priori probability. The probability of the cue being valid or invalid was $75 \%$ and $25 \%$, respectively. It was assumed that a participant's attention would be directed to the center of the cued visual quadrant. If the tactile cue was valid, then the visual target could occur at 0,90 or 180 pixels, with equal $a$ priori probability, from the center of the cued visual quadrant. If the tactile was invalid, then the visual target could occur at 350, 450, or 550 pixels, with equal a priori probability, from the center of the cued visual quadrant. It follows that in any trial, the probability for a visual target to occur at 0,90 or 180 pixels from the tactilely-cued center was $25 \%$, leading to a probability of valid cueing of $25 \% \times 3=75 \%$. The probability for a visual target to occur at 350,450 , or 550 pixels from the tactilely-cued center was $8.33 \%$, leading to a probably of invalid cueing of $8.33 \% \times 3=25 \%$.

Each trial started with a crosshair in the center of the visual display for $500 \mathrm{~ms}$. The participants were instructed to fixate at the crosshair. This was followed by the tactile cue, which lasted $60 \mathrm{~ms}$. The flicker paradigm then started with each visual scene displayed for $80 \mathrm{~ms}$ and the blank screen displayed for $200 \mathrm{~ms}$. The participants were told to click the left mouse button (without moving the mouse) as soon as they detected the flipping bar. The time elapsed from onset of the first visual frame to this moment was recorded as the response time (RT). After the mouse click, the visual scene froze and the participants were to identify the flipping bar by moving the cursor towards it and clicking the left mouse button for a second time. Erroneous data where the location of the cursor did not match that of the flipping bar was discarded ( $<1 \%$ of the total number of trials).

\subsection{Data Analysis}

The dependent variables were RT and eye-gaze data. The tactor-off data served as a baseline performance measure against which data from trials with tactile cues could be compared. The baseline RT values in the four visual target quadrants were averaged across all trials with the same visual-change quadrant. Cueing effects were determined by subtracting the RT obtained from trials with tactile cueing from the baseline RT. A positive change in RT was obtained when the baseline RT was larger than the RT with tactile cues, indicating that the participant benefited from the tactile cues by achieving 
Table I. Average RT \pm S.E. (in s) by Tactile-cue Quadrant (TQ) and Visual-target Quadrant (VQ)

\begin{tabular}{lrrrr}
\hline & \multicolumn{1}{c}{ VQ1 } & \multicolumn{1}{c}{ VQ2 } & \multicolumn{1}{c}{ VQ3 } & \multicolumn{1}{c}{ VQ4 } \\
\hline No tactile cue & $6.74 \pm 0.36$ & $6.04 \pm 0.36$ & $7.08 \pm 0.29$ & $6.37 \pm 0.25$ \\
TQ1 & $2.54 \pm 0.11$ & $9.33 \pm 0.51$ & $10.60 \pm 0.77$ & $7.82 \pm 0.41$ \\
TQ2 & $9.05 \pm 0.61$ & $2.59 \pm 0.11$ & $7.66 \pm 0.46$ & $9.96 \pm 0.75$ \\
TQ3 & $10.36 \pm 0.96$ & $8.03 \pm 0.52$ & $2.34 \pm 0.09$ & $9.84 \pm 0.58$ \\
TQ4 & $8.30 \pm 0.61$ & $9.95 \pm 0.82$ & $8.89 \pm 0.55$ & $2.28 \pm 0.08$ \\
\hline
\end{tabular}

a shorter RT. In contrast, a negative change in RT indicated that the participant slowed down (i.e., a longer RT). From the four possible tactile-cue locations on the participant's back and the four possible visual-change quadrants on the computer screen, there were a total of sixteen (16) tactile-cue / visualchange quadrant pairs. Of the sixteen (16) pairs, four (4) corresponded to trials with valid tactile cues, and twelve (12) with invalid tactile cues. Data from each participant was processed separately.

The eye-gaze data provided a means to observe and measure participants' overt visual spatial attention. Generally speaking, a spatial attentional shift does not necessarily require a change in eye-gaze direction (e.g., a covert shift of attention can occur without a gaze change). However, for the present study, in which a visual search task on a large display was conducted, it was reasonable to use eye-gaze data to locate a participant's visual attention. Specifically, the present study used the eye-gaze data to estimate initial saccades to each visual quadrant. The initial saccades were measured by identifying the quadrant that the participant looked at after the start of each trial. For example, if the participant's first glance was toward the upper-right quadrant (VQ1), then we expected to see the eye-gaze location move from the center of the visual display towards the right and up. One initial saccade would then be counted for VQ1. Specifically, the initial saccades were defined to be the first eye-gaze data that lay outside a visual angle of $\pm 1.53^{\circ}$ (or equivalently, outside a central area of $3.6 \mathrm{~cm} \times 3.6 \mathrm{~cm}$ ) from the fixation crosshair at the center of the visual display.

\section{RESULTS}

\subsection{Mean Response Time}

The average RT data and its standard errors are listed in Table I by tactile-cue quadrants (no cue, and TQ1-TQ4) and visual-target quadrants (VQ1-VQ4). It appears that average RT for the baseline condition of no tactile cueing was the shortest for VQ2 (6.04 s) and the longest for VQ3 (7.08 s), but a repeated-measures (RM) ANOVA with the factor visual quadrant showed that visual quadrant was not a significant factor $[F(3,27)=1.28$, Huynh-Feldt $p=0.303]$.

The changes in RT for valid tactile cueing (i.e., for cue-target distances of 0,90 , and 180 pixels), averaged over all ten participants, are shown in Figure 3. As expected, the values in Figure 3 were all positive, indicating a strong cueing effect for which the participants' RT decreased when given valid tactile cues. Results from one-sample $t$-tests confirmed that all values in Figure 3 are significantly different from zero (i.e., changes in RT are significant due to valid tactile cueing).

The existence of a visual attentional gradient was determined by assessing whether there was a decreasing trend in the change in RT as the distance between the visual target and the center of attention grew larger. Inspection of Figure 3 indicates a decreasing trend as cue-target distance increased for each TQ/VQ pair, suggesting that the benefits due to valid tactile cueing waned as the visual targets moved further away from the center of the cued quadrant. The trend provided evidence for a visual gradient of attention. A two-way within-subject RM-ANOVA with the factors TQ (or VQ) and cue-target Distance showed that the effect of TQ (or VQ) was not statistically significant, but that cuetarget Distance was a significant factor for changes in RT when the tactilely-cued quadrant matched 


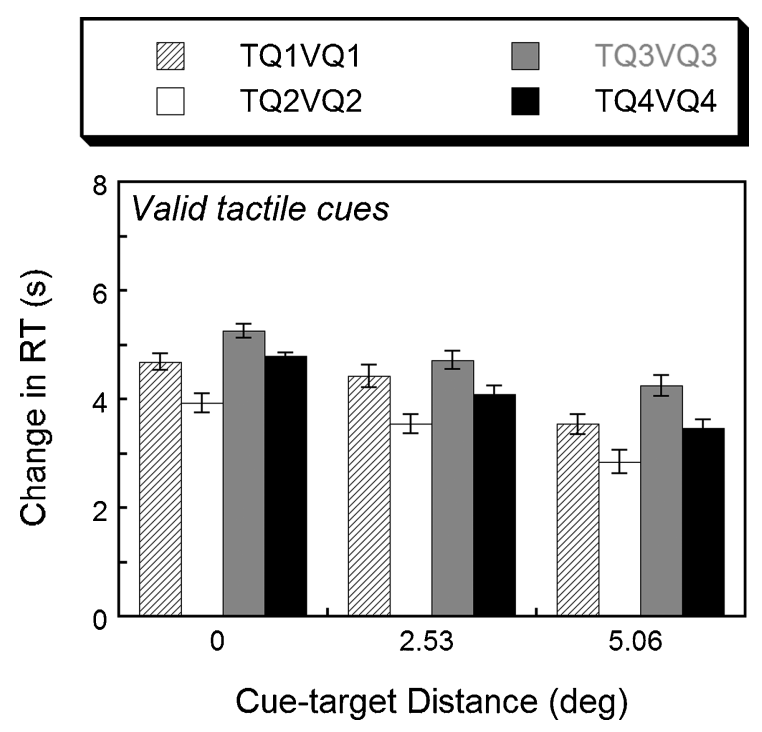

Fig. 3. Change in RT for valid tactile-cueing condition as a function of cue-target distance in visual angle. Shown are the averages and standard errors. Note that with valid cueing, the tactile quadrants TQ always match the visual quadrants VQ.

the visual-change quadrant (TQ: $[F(3,27)=1.90$; Huynh-Feldt $p=0.162$; Distance: $[F(2,18)=25.26$, Huynh-Feldt $p<0.001)$. The interaction between TQ and Distance was not significant $(F(6,54)=$ 0.269 , Huynh-Feldt $p=0.920$ ), which was consistent with the observation that, for each TQ/VQ pair, changes in RT decreased as cue-target distance increased. A Bonferroni multiple comparisons test further indicated that the changes in RT at the three cue-target distances were significantly different from one another (mean \pm S.E. in s: $4.6 \pm 0.4$ (0 pixel); $4.1 \pm 0.4(90$ pixels $) ; 3.5 \pm 0.4(180$ pixels $)$ ).

In contrast, the changes in RT from trials with invalid tactile cues (not shown graphically, but see the listing in Table I) were zero or negative, suggesting that RT remained the same or increased when participants were given invalid tactile cues, respectively. Results of $t$-tests showed that the changes in RT for 14 out of the 36 (12 nonmatching TQ/VQ pairs $\times 3$ cue-target distances) invalid-cueing cases were significantly different from zero, indicating that, 39\% of the time, changes in RT were significant when tactile cues were invalid. The remaining changes in RT were either not significantly different or marginally different from zero. A one-way within-subject RM-ANOVA found cue-target Distance to be significant for changes in RT $(F(2,18)=11.15$, Huynh-Feldt $p=0.001)$. A subsequent Bonferroni multiple comparisons test indicated two groups of data: 350 pixels $(-1.2 \pm 0.3 \mathrm{~s})$, and 450 and 550 pixels $(-3.3 \pm 0.5 \mathrm{~s}$ and $-3.2 \pm 0.6 \mathrm{~s}$, respectively).

To better visualize the visual attentional gradient effect due to tactile cueing, the changes in RT averaged over all trials with the same cue-target distance are shown in Figure 4 for both valid and invalid cueing. As expected, there are two groups of data points, one for valid cueing with positive changes in RT (or equivalently, with decreases in RT, at cue-target distances of 0,90 and 180 pixels) and the other for invalid cueing with negative changes in RT (cue-target distances of 350, 450, and 550 pixels). By visual inspection, the change in RT monotonically decreased as distance grew, indicating the existence of gradient of visual attention due to tactile cueing. To quantify this data, two straight lines were fit to the valid-cue and invalid-cue data, respectively (see Figure 4). The linear fit for valid cueing was marginally significant $(F(1,28)=3.98, p=0.0557)$ and the linear fit for invalid cueing was significant $(F(1,28)=6.73, p=0.0149)$. The significantly higher intercept value for valid trials $(4.67 \mathrm{~s})$ compared to that for invalid trials $(1.88 \mathrm{~s}$ ) was consistent with the expectation of speeding-up of 


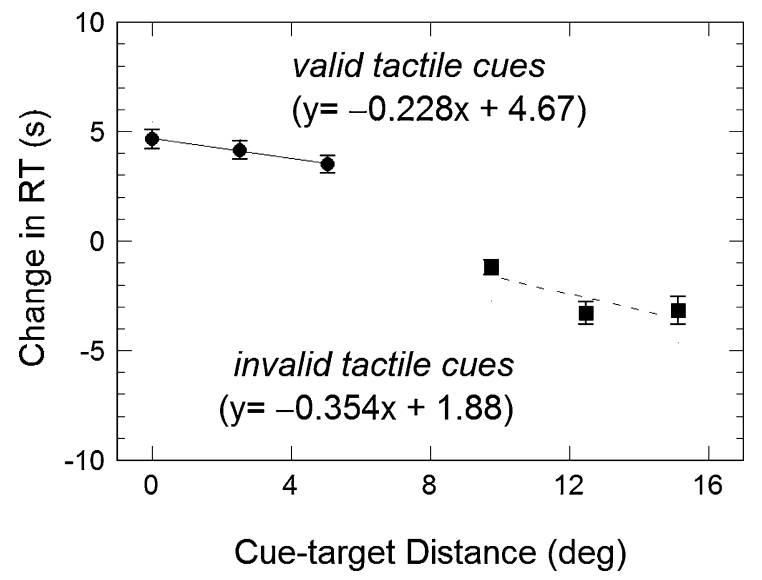

Fig. 4. Change in RT as a function of cue-target distance in visual angles for both valid and invalid cueing conditions. Shown are the averages and standard errors over all trials with the same cue-target distance. Also shown are the linear fitting lines for the valid and invalid cueing conditions.

visual search following valid tactile cues and slowing down of visual search following invalid tactile cues, respectively. The results of a three-way within-subject RM-ANOVA confirmed that Valid/Invalid condition (i.e., TQ/VQ pairing) $(F(1,9)=256.01, p<0.001)$ and cue-target Distance $(F(2,18)=24.77$, $p<0.001)$ were significant factors for changes in RT, but not TQ $(F(3,27)=0.08, p=0.97)$.

\subsection{Eye-Gaze Data}

The number of initial saccades, averaged across all participants for the baseline condition of no tactile cueing, were (mean \pm S.E.) $34 \pm 1.4,59 \pm 1.3,13 \pm 0.7$, and $13 \pm 1.3$ when visual targets occurred in quadrants VQ1, VQ2, VQ3, and VQ4, respectively. On average, the upper-left quadrant VQ2 received the most initial saccades (59), followed by the upper-right quadrant VQ1 (34). These numbers were significantly larger than the number of average initial saccades received by the lower-left quadrant VQ3 (13) or the lower-right quadrant VQ4 (13). The fact that VQ2 received the most initial saccades was consistent with the nonsignificant tendency for the baseline RT for VQ2 to be the shortest (see Table I). However, the significantly lower initial saccade counts for VQ3 and VQ4 did not manifest themselves in significantly higher baseline RTs for these two quadrants (see Figure 3).

The numbers of initial saccades averaged across all participants for each TQ/VQ pair are shown in Figure 5. There were significantly larger numbers of initial saccades for the 4 valid-cue cases, where the tactilely-cued quadrant matched the visual-change quadrant, than those for the 12 invalid-cue cases. The larger number of initial saccades for the valid cueing conditions corresponded to the positive changes in RT shown in Figures 3 and 4. Together, the results from Figures 3, 4, and 5 imply that with valid tactile cueing, overt visual attention was directed towards the quadrant of visual change, which led to a significant decrease in RT to find the change in that quadrant.

\section{DISCUSSION}

The results of the present study confirmed-for trials with valid and invalid tactile cues-a significant increase in RT when the distance between the center of the cued quadrant and the visual target increased, thereby demonstrating an attention gradient effect. In addition, eye-gaze data indicated that the majority of the initial saccades went into the visual quadrant that corresponded to the tactilely-cued quadrant, thereby demonstrating an overt shift of visual attention due to tactile 


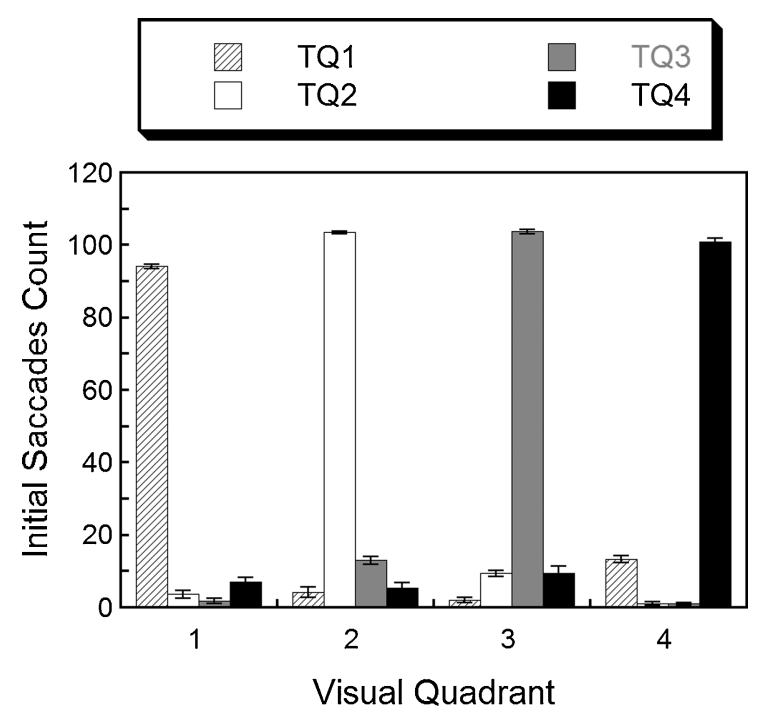

Fig. 5. Number of initial saccades averaged over all participants for both valid and invalid cueing conditions. Shown are the averages and standard errors over all trials with the same TQ/VQ pair.

spatial cues. This data is consistent with the finding that RT, to find a visual-change target, decreased with valid tactile cues and increased (or did not change significantly) due to invalid tactile cues.

These results mirror the findings of similar studies related to tactile cueing of visual spatial attention, although the RTs measured in the present study were much longer than those obtained in our earlier work (e.g., Jones et al. [2008]). The difference in RT results may be explained by the greater difficulty or mental workload of the visual search task used in the present study, where participants needed to search for one flipping bar among 40 bars, as opposed to one in 12 bars in Jones et al.'s [2008] study. It has been reported that task performance deteriorates (in our case, RT increases) when mental workload is too high or too low (e.g., Proctor and Van Zandt [2008]). Post-experiment comments from the participants indicated that they found the visual-search task in the present study to be demanding and to require a lot of concentration.

The tactile cues in the present study differed from some of our earlier ones, in that the cues and targets were not collocated in space. The proximal-distal cueing effect observed was most likely largely endogenous in that the participants had to make a strategic shift in their (overt) visual attention upon receiving a tactile cue on their backs (see Spence and Driver [2004]; Wright and Ward [2008]). The relationship between the four cue locations on the back and the four visual quadrants where targets could occur was, however, a straightforward, spatially compatible one [Proctor and Vu 2006], as was evidenced by the strong cueing effect observed with the participants in the this study, who received little practice with the mapping prior to data collection. In many practical applications such as an automobile collision-warning system, the use of proximal cues for distal events is more appropriate, since it would not be feasible to implement cues at the exact locations of impending collisions. A potential disadvantage of such cues is that a user of a warning system can "tune out" such signals when instructed to (see Jones et al. [2008]) or when the signals are perceived to be false or annoying (see Wright and Ward [2008], p. 24).

A debate in the attention literature is whether spatial attention moves in an analogue or discrete/quantal manner, with contradictory evidence supporting either theory (e.g., Shioiri et al. [2002], for analog [Sperling and Weischelgartner 1995], for quantal/multiple fixed gradients). The results of 


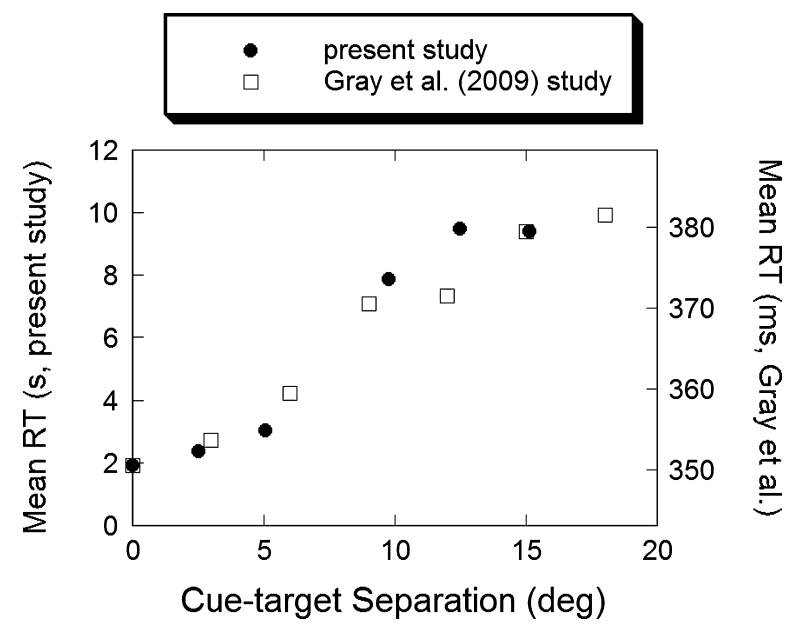

Fig. 6. Comparison of attention gradient effects from the present study (open circles) and Gray et al.'s [2009] study (open squares).

the present study seem to support the notion that visual spatial attention shifted continuously across the visual field, for two reasons. First, the observed RT grew linearly with respect to the cue-target distance. This linear relationship could presumably imply a constant velocity of attentional shift, which in turn supports previous findings of analog attentional shifts (see Wright and Ward [2008], for a detailed summary). Second, a replay of eye-gaze data superimposed on the alternating visual scenes revealed a continuous mini-search pattern within each visual quadrant where participants searched within a small area that was progressively further away from the center of the tactilely-cued visual quadrant. The pattern was prevalent among all participants, and differed only in the quadrants where each participant started and ended the search, clockwise vs. counterclockwise trajectory, and the speed of eye-movements.

The central finding of the present study, namely the gradient of attention for proximal-distal tactile cueing of visual spatial attention, can be quantitatively compared to that of our earlier study using (almost) collocated tactile cue and visual target [Gray et al. 2009]. Data from both studies is shown in Figure 6. The open circles are the mean RTs from the present study. The open squares are from Gray et al.'s study [2009, Figure 3] for the tactile cueing condition where vibrotactile cues were presented on the forearm and visual targets were presented along the sides of the forearm at the same visual angles (with the forearm placed parallel to the torso in front of the participant). The mean RT data from Gray et al. [2009] was scaled and shifted so that the range matched that of the mean RTs from the present study for cue-target separations up to $15^{\circ}$. There is a striking similarity between the two sets of data, despite major differences in the methods of the two studies (proximal-distal vs. proximalproximal cueing, noncollocated vs. collocated cue and target, tactile cues on the back as opposed to on the forearm, spatially-informative vs. spatially-noninformative cues, visual change detection vs. nearfar discrimination task). This similarity suggests that visual spatial attention is (re-)oriented in the same manner whether tactile cues are presented in very different locations from the visual targets (present study) or in locations next to the targets [Gray et al. 2009]. Furthermore, the similar findings provide further evidence that tactile cues can be effectively deployed when they are presented in a very different location (e.g., the driver's body) than the critical target (e.g., an incoming car). This is important because it provides more flexibility for interface designers in terms of the placement of cueing signals. 
Care should be taken when utilizing the results of the present study for the design of crossmodal cueing systems for practical applications. A potential limitation of this study may stem from the use of a chin rest with a forehead stabilizer. It has been shown that tactile localization can be affected by both head orientation [Ho and Spence 2007] and eye-gaze direction [Harrar and Harris 2009]. The results of the present study, therefore, may be affected when participants are free to move their heads and eyes. Another potential limitation of the present study concerns the assumption that the center of visual attention due to tactile cueing is located at the center of the cued quadrant. This assumption cannot be easily confirmed in this study. Given the asymmetry in the number of initial saccades with regard to visual quadrants, it is conceivable that there may be a bias in eye movement that results in visual attention being more central in one quadrant than in another. This would have affected the results shown in Figure 4 by introducing noise into the calculation of cue-target distances.

Future work will proceed in several directions. First, it will be interesting to process the massive amount of eye-gaze data and model the various visual search patterns employed by the participants. The goal will be to identify the relevant parameters contributing to the observed cueing effects. Second, experiments will be conducted with a finer division of the visual screen by using a more densely-packed tactor array (e.g., 3-by-3). The challenge will be to balance the possible stronger cueing effects (due to the decreased cue-target distance with valid tactile cues) with the ambiguity in the localization of the tactile cues that might arise. Finally, research should be conducted in a more ecologically-valid setting, such as a driving simulator, to confirm the benefits of tactile cueing of visual attention in practical applications. A quantitative assessment of the magnitude of cueing effects (in terms of the reduction in RT) in a realistic driving simulator will provide the data needed for interface designers to evaluate whether a proposed collision warning system will result in enough lead time for collisions to be avoided and lives to be saved.

\section{REFERENCES}

Assad, J. A. 2003. Neural coding of behavioral relevance in parietal cortex. Current Opinion Neurobiol. 13, $194-197$.

BREfCZYNSKI, J. A. AND Deyoe, E. A. 1999. A physiological correlate of the spotlight of visual attention. Nature NeuroScience 2, 370-374.

Downing, C. J. And Pinker, S. 1985. The spatial structure of visual attention. In Attention and Performance Vol. 11, M. I. Posner and O. S. M. Marin Eds., Erlbaum, Hillsdale, NJ, 171-188.

Driver, J. AND Spence, C. 1998. Cross-modal links in spatial attention. Philosoph. Trans. Royal Soc. London Series B-Biolog. Sci. $353,1319-1331$.

FöCKer, J., Hötting, K., Gondan, M., AND RöDER, B. 2010. Unimodal and crossmodal gradients of spatial attention: Evidence from event-related potentials. Brain Topogr. 23, 1-13.

Gray, R., MohebBi, R., AND TAN, H. Z. 2009. The spatial resolution of crossmodal attention: Implications for the design of multimodal interfaces. ACM Trans. Appl. Perception 6, 1, Article 4.

GRAY, R. AND TAN, H. Z. 2002. Dynamic and predictive links between touch and vision. Exper. Brain Res. 145, 50-55.

HARRAR, V. AND HARRIS, L. R. 2009. Eye position affects the perceived location of touch. Exper. Brain Res. 198, 403-410.

Ho, C. ANd Spence, C. 2007. Head orientation biases tactile localization. Brain Res. 1144, 136-141.

Ho, C., TAN, H. Z., AND Spence, C. 2005. Using spatial vibrotactile cues to direct visual attention in driving scenes. Transport. Res. Part F: Traffic Psychol. Behav. 8, 397-412.

Jones, C. M., Gray, R., Spence, C., And Tan, H. Z. 2008. Directing visual attention with spatially informative and spatially noninformative tactile cues. Exper. Brain Res. 186, 4, 659-669.

McMains, S. A. AND Somers, D. C. 2004. Multiple spotlights of attentional selection in human visual cortex. Neuron 42,677-686.

McMains, S. A. AND Somers, D. C. 2005. Processing efficiency of divided spatial attention mechanisms in human visual cortex. J. Neuroscience 25, 9444-9448.

Perrott, D. R., SABeri, K., Brown, K., And Strybel, T. Z. 1990. Auditory psychomotor coordination and visual search performance. Percep. Psychophysics 48, 214-226.

Posner M. I., Snyder C. R. R., AND Davidson B. J. 1980. Attention and the detection of signals. J. Exper. Psych. General 109, 2 , $160-174$. 
Proctor, R. W. and Read, L. E. (EDS.) 2009. Attention Vol. 2: Visual Attention. Sage, London.

Proctor, R. W. And Van Zandt, T. 2008. Human Factors in Simple and Complex Systems 2nd Ed. CRC Press, Boca Raton, FL.

Proctor, R. W. ANd Vu, K.-P. L. 2006. Stimulus-Response Compatibility Principles: Data, Theory, and Application. CRC Press, Boca Raton, FL.

Rensink, R. A. 2000. Visual search for change: A probe into the nature of attentional processing. Visual Cognition 7, 345-376.

Schmitt, M., Postma, A., AND DE HAAN, E. H. F. 2001. Cross-modal exogenous attention and distance effects in vision and hearing. Euro. J. Cognitive Psych. 13, 343-368.

ShePherd, M. AND MÜLler, H. J. 1989. Movement versus focusing of visual attention. Percep. Psychophysics 46, $146-154$.

Shioiri, S., YAmamoto, K., KageYama, Y., AND Yaguchi, H. 2002. Smooth shifts of visual attention. Vision Research 42, $2811-2816$.

Shulman, G. L., Remington, R. W., And Mclean, J. P. 1979. Moving attention through visual space. J. Exper. Psych. Human Percep. Perform. 5, 522-526.

Somers, D. C., Dale, A. M., Seiffert, A. E., ANd Tootell, R. B. H. 1999. Functional MRI reveals spatially specific attentional modulation in human primary visual cortex. In Proc. US Nat. Acad. Science 96, 4, 1663-1668.

Spence, C. And Driver, J. 2004. Crossmodal Space and Crossmodal Attention. Oxford University Press, Oxford, UK.

Spence, C., Nicholls, M. E. R., Gillespie, N., AND Driver, J. 1998. Cross-modal links in exogenous covert spatial orienting between touch, audition, and vision. Percep. Psychophysics 60, 544-557.

Sperling, G. And Weichselgartber, E. 1995. Episodic theory of the dynamics of spatial attention. Psych. Rev. 102, 503-532.

Tan, H. Z., Gray, R., Spence, C., Jones, C. M., AND Mohd Rosli, R. 2009. The haptic cuing of visual spatial attention: Evidence of a spotlight effect. In Proceedings of SPIE-IS\&T Electronic Imaging, Human Vision and Electronic Imaging XIV, B. E. Rogowitz and T. N. Pappas Eds., SPIE, vol. 7240-72400I.

TAN, H. Z., Gray, R., Young, J. J., AND Traylor, R. 2003. A haptic back display for attentional and directional cueing. Haptics: Electron. J. Haptics Res. 3.

TREUE, S. 2001. Neural correlates of attention in primate visual cortex. Trends Neuroscience 24, 295-300.

Van Der Burg, E., Olivers, C. N. L., Bronkhorst, A. W., ANd Theeuwes, J. 2009. Poke and pop: Tactile-visual synchrony increases visual saliency. Neuroscience Lett. 450, 60-64.

VAn Der Heijden, A. H. C. 1992. Selective Attention in Vision. Routledge, London.

Van Erp, J. B. F., Van Veen, H. A. H. C., Jansen, C., AND Dobbins, T. 2005. Waypoint navigation with a vibrotactile waist belt. ACM Trans. Appl. Percept. 2, 106-117.

WRight, R. D. AND WARD, L. M. 2008. Orienting of Attention. Oxford University Press, Oxford, UK.

Received June 2009; revised August 2010; accepted October 2010 